\author{
Accelerator Division \\ Alternating Gradient Synchrotron Department \\ BROOKHAVEN NATIONAL LABORATORY \\ Associated Universities, Inc. \\ Upton, New York 11973
}

Accelerator Division

Technical Note

AGS/AD/Tech. Note No. 323

SIMULATING THYRISTOR CONVERTER

S.Y. Zhang

August 1, 1989 


\section{Simulating Thyristor Converter}

\section{Introduction}

The controlled thyristor converter, also called the multiphase rectifier, of ten. causes problems in AC-DC power conversion system analysis. The reason is that the converter is a nonlinear, nonuniformly sampled discrete system. with marginal sampling rates, and therefore an analytical solution to the converter analysis is always accompanied with considerable errors. In literature the converter is often simplified as a constant amplifier, a phase delay factor, a time delay factor, or a first or second order Pade approximations to the time delay factor, etc. If the voltage regulator time constant, or for the current regulations the load time constant, is comfortably long compared to the sampling period, then such models can be used. In many occasions, this is not the case. For example in a pulsed power conversion system, the whole process of a cycle completes in a few or scores of samplings. The analysis error caused in using approximate models may not be negligible. Furthermore, if subharmonic ripples and harmonic ripples are concerned, then these models becomes totally useless. Therefore, a simulation to the thyristor converter will be of interest in the power system design and analysis.

In this note we present simulations to the thyristor converters. The simulations are written by MATLAB on IBM PC's in recursive procedures. At present only 8188 memory elements are in use, if the memory can be extended then the simulation resolution can be improved, or with the same resolution the simulating time can be extended. The simulations are presented in three packages. First package concerns the converter itself. Second package concerns the converter with commutations, and the last package concerns the regulated converters, either by voltage or current, with possible commutations. The last one is more comprehensive and therefore is more useful in system analyses.

\section{Thyristor Converter Simulation}

In the thyristor converter simulation, we adopt the ramp comparator firing principle. Although there are various versions of the firing system including analog, digital, and analog with digital ones, the ramp comparator still functions as the fundamental firing principle without loss of generality.

The simulations are based on a 3 phase AC power supply system, and the parameters in the simulation can be listed as the follows.

1. Number of pulse, $n$. For instance, in Booster we use two 12 pulse systems to constitute a 24 pulse converter system. The system can be simulated by either the combination of the two 12 pulse systems or a one 24 pulse system.

2. Amplitude of the bridge sine waveform for each phase or each pulse, $a a(i)$. For example the 4th pulse is produced from the bridge waveform that is with an amplitude of $a a(4)$. This provides convenience to simulate the phase imbalance and other power line disturbances.

3. Simulation time steps and period. We use tini to represent the initial simulation time, tint the time interval, and $t f$ in the final time. It is possible to define nonuniform simulation time intervals, but we use the uniform one in the present simulation. The simulation steps are constrained by the memory, at present we take 800 to 2000 steps for simulations, that is denoted as tnum. 
4. Fundamental Frequency, $\omega$. For most power systems we simply let $\omega=2 \pi \times 60$.

5 . Firing range, $f r$. For instance we let $f r=\pi$, and this is good for full range conversion and energy invert.

6. Ramp slope, rs. This determines the gain of the converter, and also it is related to the firing range. In fact we have $r s=\frac{-2 \omega \times f r}{\pi \times 2 \pi}$.

7. Ramp position, trp. For each converter system, the trp is unique, and it should be placed between 0 to $\frac{2 \pi}{\omega \times n}$.

8. Reference, $u$. This is the waveform shown on the ramp comparator and should be defined by users.

With all these parameters defined, the output of the thyristor converter can be calculated and plotted. In the following we show an example. We let the pulse number be 24, the amplitudes be 1000 for all three phases and therefore also for all pulses. The simulation time is from 0 to $20 \mathrm{~ms}$ with each step of $0.025 \mathrm{~ms}$, and the total simulation steps be 800 . The fundamental frequency is $60 \mathrm{~Hz}$. Let the firing angle be 180 degrees, and therefore the ramp slope be -240 . The ramp position is placed at $\frac{\pi}{\omega \times n}=0.3472 \mathrm{~ms}$. Then, for different references we show the converter voltage output waveforms in Fig.1. The nonlinearity of the converter is clearly shown in the plots. Since the reference is proportional to the firing angle, it is not linearly proportional to the DC voltage level.

With merely the number of pulses changed to 12 and 6 , the simulation plots the converter output waveforms for the first reference waveform in Fig.1. These plots are shown in Fig.2.

\section{Thyristor Converter with Commutations}

The commutation is an important problem in thyristor power conversions, since it affects the system power factor, the voltage ripple, and causes additional delay in the regulation loops. There are some formulae concerned about the analytical solutions of the commutations. Since the commutation is determined by many factors, these formulae are conditional and therefore only good for estimations.

Taking advantage of the computing, we use prevalent conditions in the simulation to determine the commutation. For example, for every step we calculate the load current timingly and use it along with the bridge voltage to calculate the commutation. Thus, possible errors from the converter output current and voltage estimations can be avoided. The principle of the commutation computing is shown in Fig.3. We assume that thyristor 1 was on and thyristor 2 is just triggered. The commutation process is then started. We assume that there is a loop current shown in the schematic that is growing and is governed by the loop voltage and the transformer leakage inductance and line inductance. The loop voltage is the difference of the two line voltages and the line inductances can be found from the transformer data and circuit estimations. Once the loop current reaches the load current, the commutation process completes. In the program, we have overlooked the voltage drop on thyristors, the resistances on the circuit, and other factors. If necessary, these factors can be easily installed in the program.

The parameter necessary in the converter with commutation simulation is therefore only the load transfer function, and the bridge line inductances. 
In the example, in addition to the parameters shown in the last section, we assume that the load is a magnet with an inductance of $200 \mathrm{mH}$ and a resistance of $1 m \Omega$, that has a transfer function $\frac{200}{s+5}$. The inductance for each power line is assumed to be $20 \mu H$. The converter voltage output is then plotted in Fig.4.1 where the current and the voltage reference are also plotted. In Fig.4.2, we show the waveform of the converter with $40 \mu H$ power line inductance. In Fig.4.3, we show the waveform of a 12 pulse converter.

\section{Regulated Converter}

It is usually difficult to simulate fast dynamic affairs in a PC. However for this particular problem, there exists a simple solution. It is interesting to notice that after firing the converter voltage waveform is simply determined by the bridge sine waveform, until the next firing. This provides considerable convenience in the converter regulation simulation. Consider only the voltage regulation with a unity feedback coefficient. The present converter voltage waveform is used to compare with the reference and then to drive the regulator. The output of the regulator is then compared with the ramp and determine the next firing. Due to the above mentioned convenience, the simulation is in fact executed periodically alike an open loop calculation that saves significant computations and therefore gives rise a possibility to realize the simulation.

The parameters necessary for the regulated converter simulation is of course the transfer function of the regulator, and if any, the transfer function of the voltage divider and filters. It is ready to include the current regulation and other configurations.

In the example, we show the regulated 24 pulse system under a unity voltage feedback and the regulator

$$
C(s)=\frac{0.5}{s+40}
$$

This transfer function implies a regulator with a bandwidth of $6.36 \mathrm{~Hz}$ and a total DC loop gain roughly speaking at 12.5 . The regulated voltage waveform is shown in Fig.5.1. In Fig.5.2 and Fig.5.3, we show the waveforms that are corresponding to the loop gains 37.5 and 75 , respectively. This is done by only modifying the numerator of the regulator from 0.5 to 1.5 and 3 , respectively.

It is easy to observe the differences between the performances of the regulated and unregulated systems, that are shown in Fig.5.1 and Fig.1, respectively. Also it is easy to observe the differences of the performances of regulated converters with different loop gains, see Figs. 5.1, 5.2 and 5.3. 


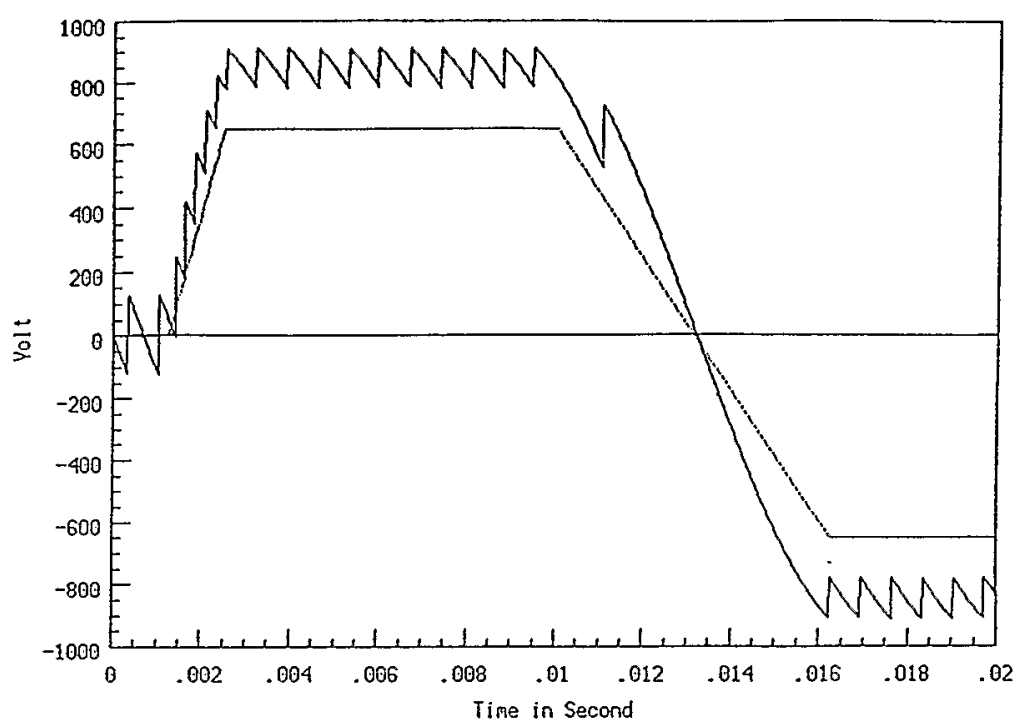

Fig.1.1

24 pulse converter with normalized reference

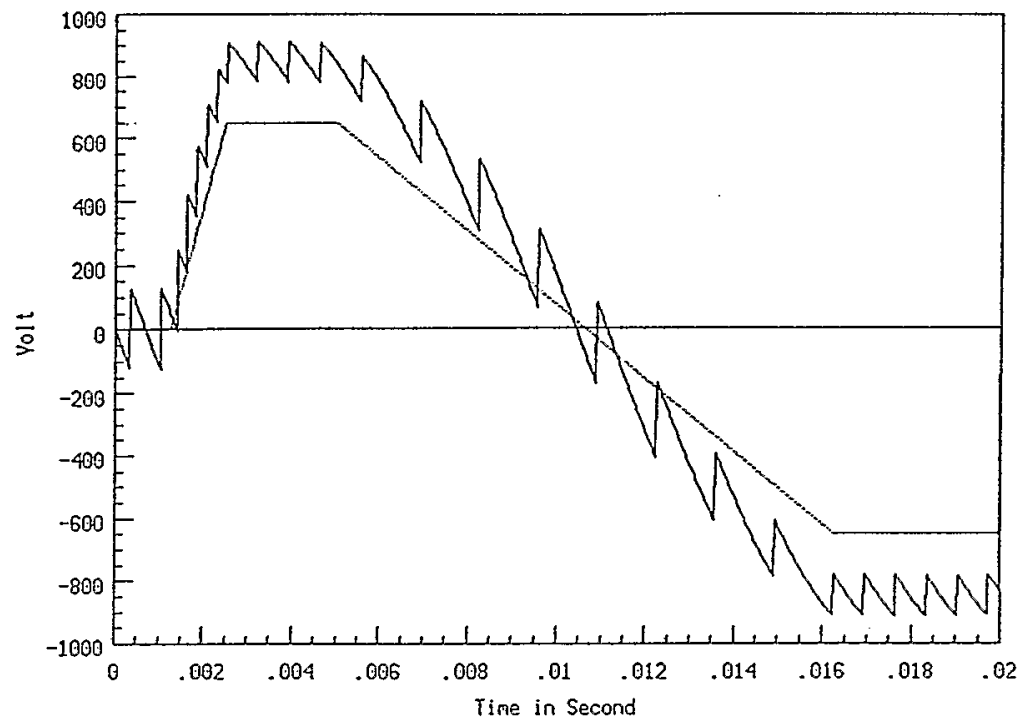

Fig.1.2

24 pulse converter with normalized reference

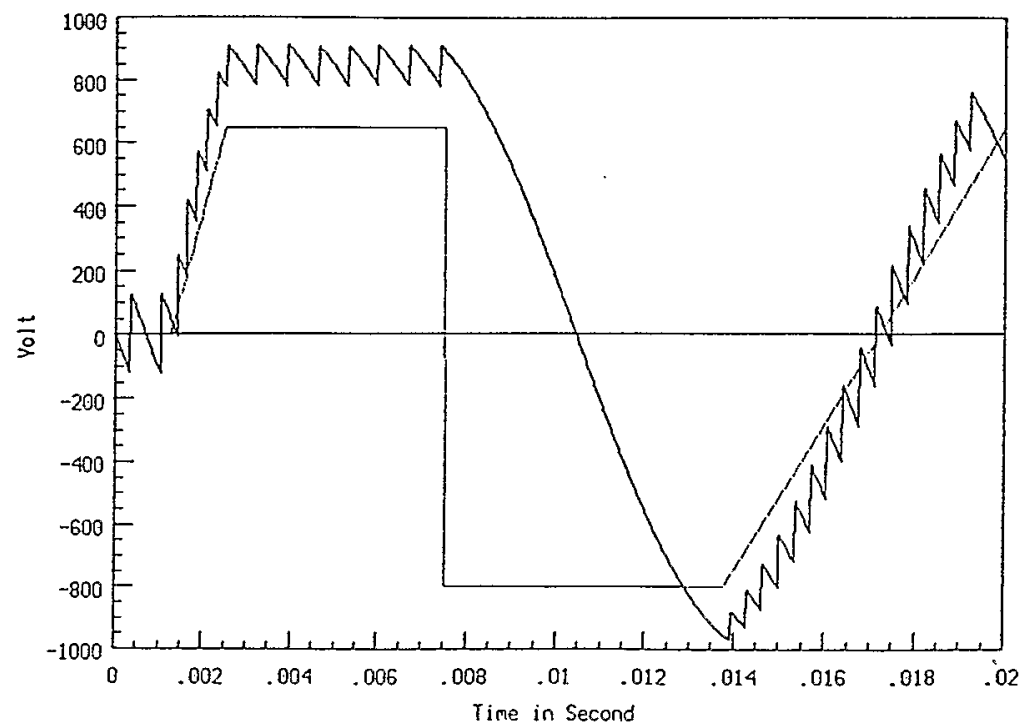

Fig. 1.3

24 pulse converter with normalized reference 


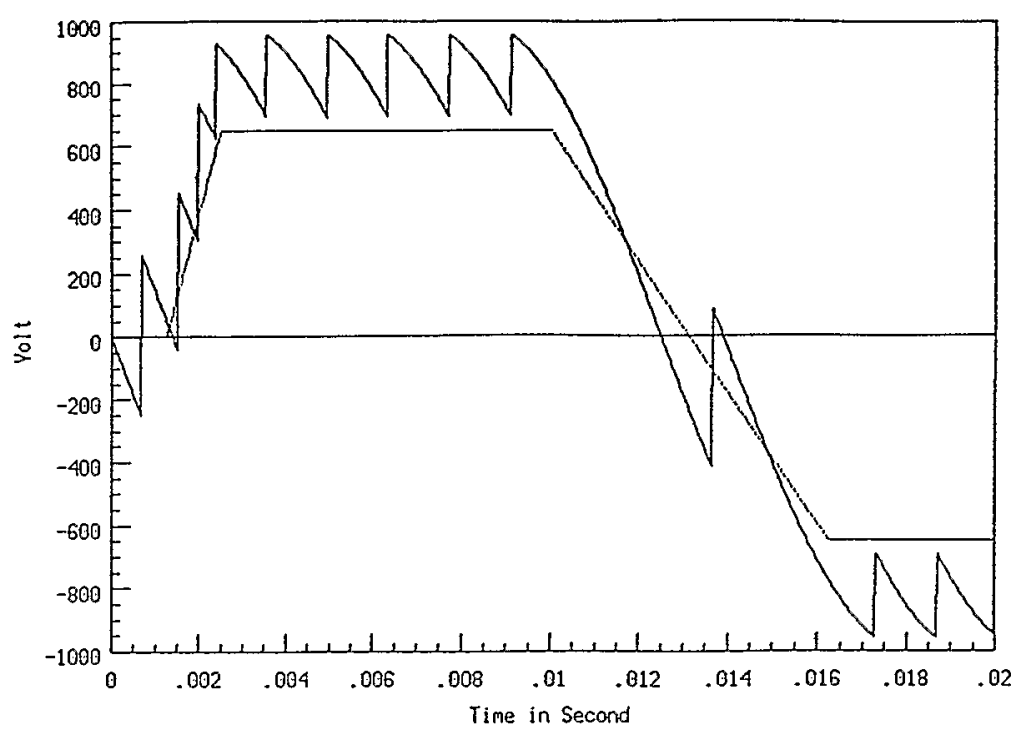

Fig. 2.1

12 pulse converter with normalized reference

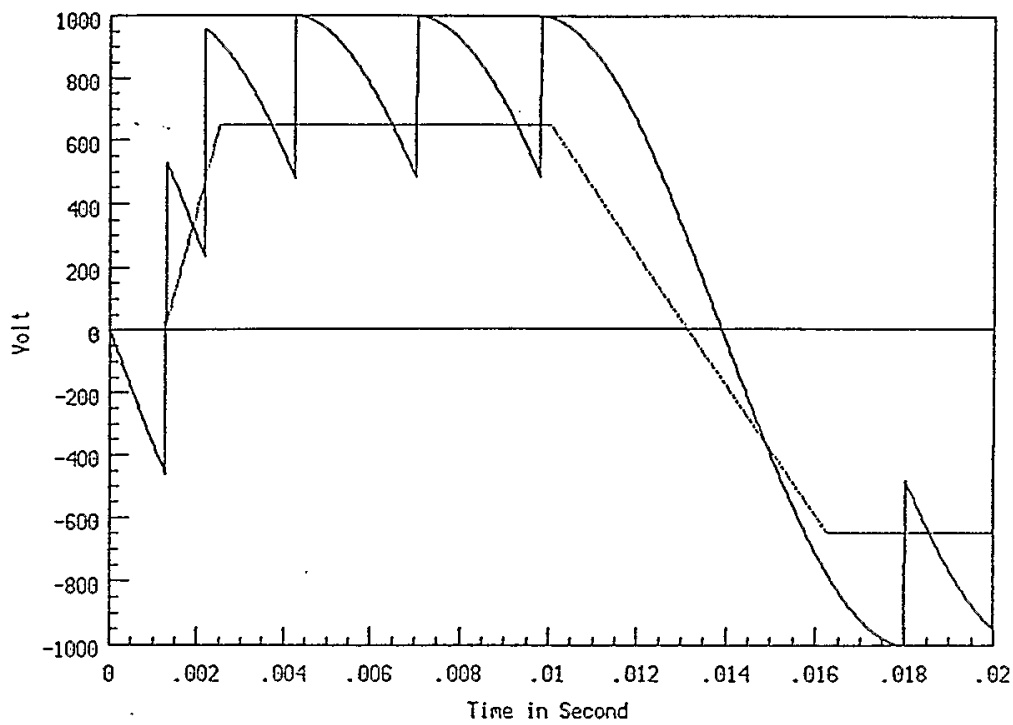

Fig. 2.2

6 pulse converter with normalized reference

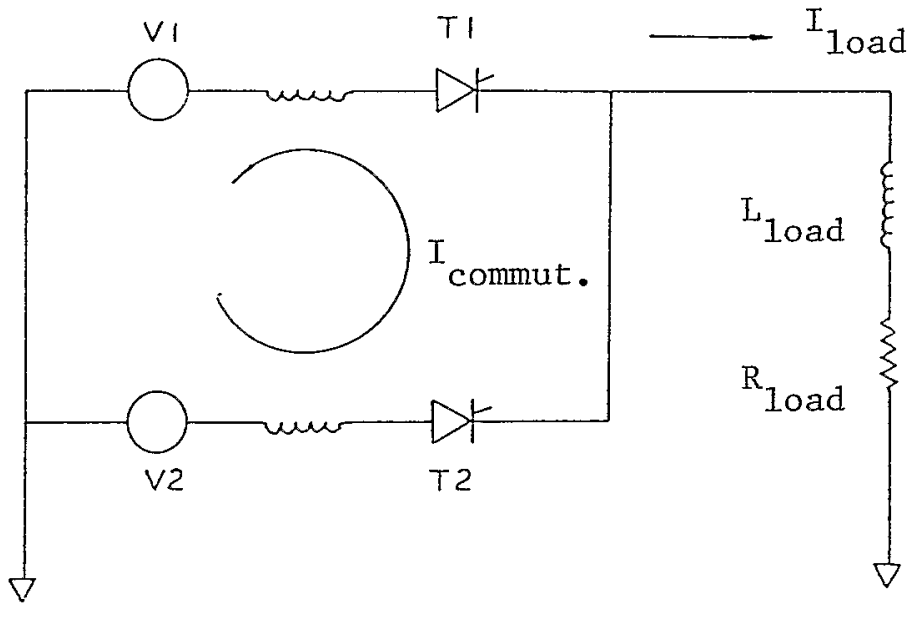

Fig. 3

Commutation diagram 


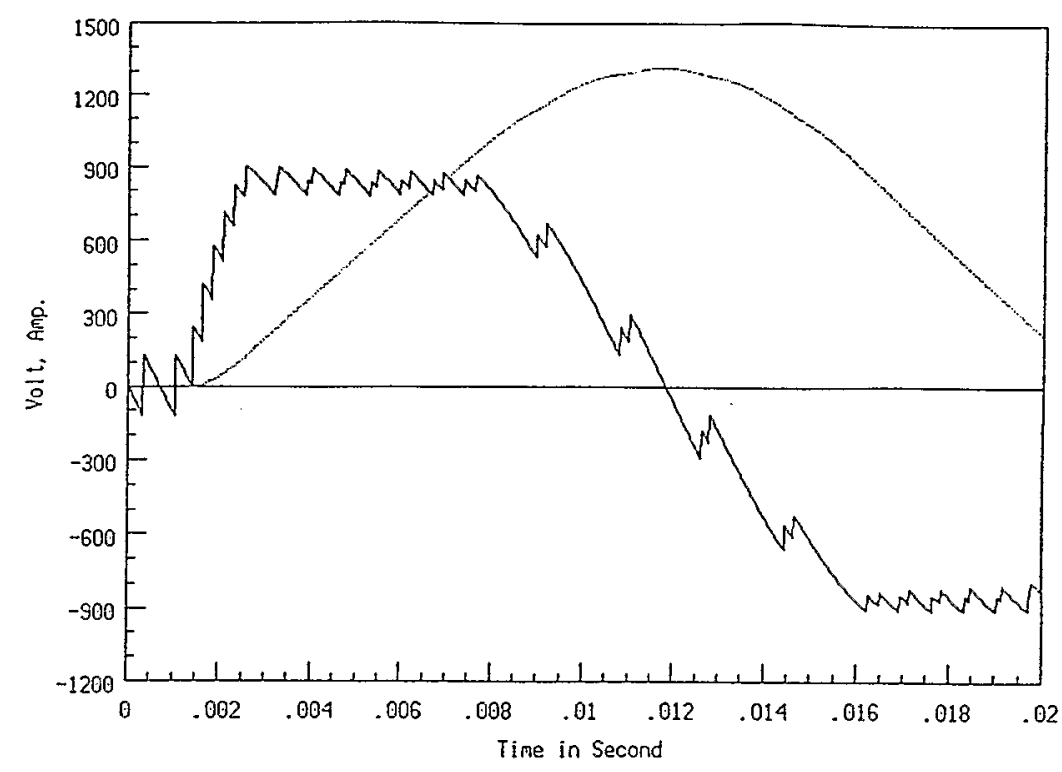

Load Current

Fig.4.1

24 pulse converter with commutation

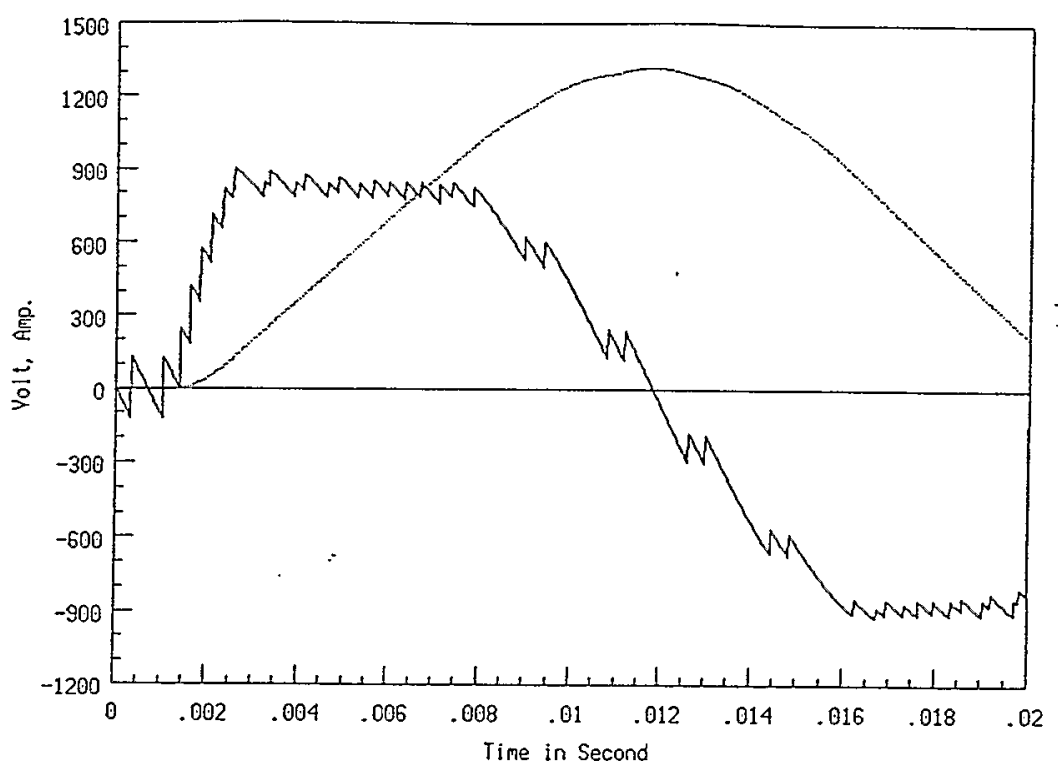

Load Current

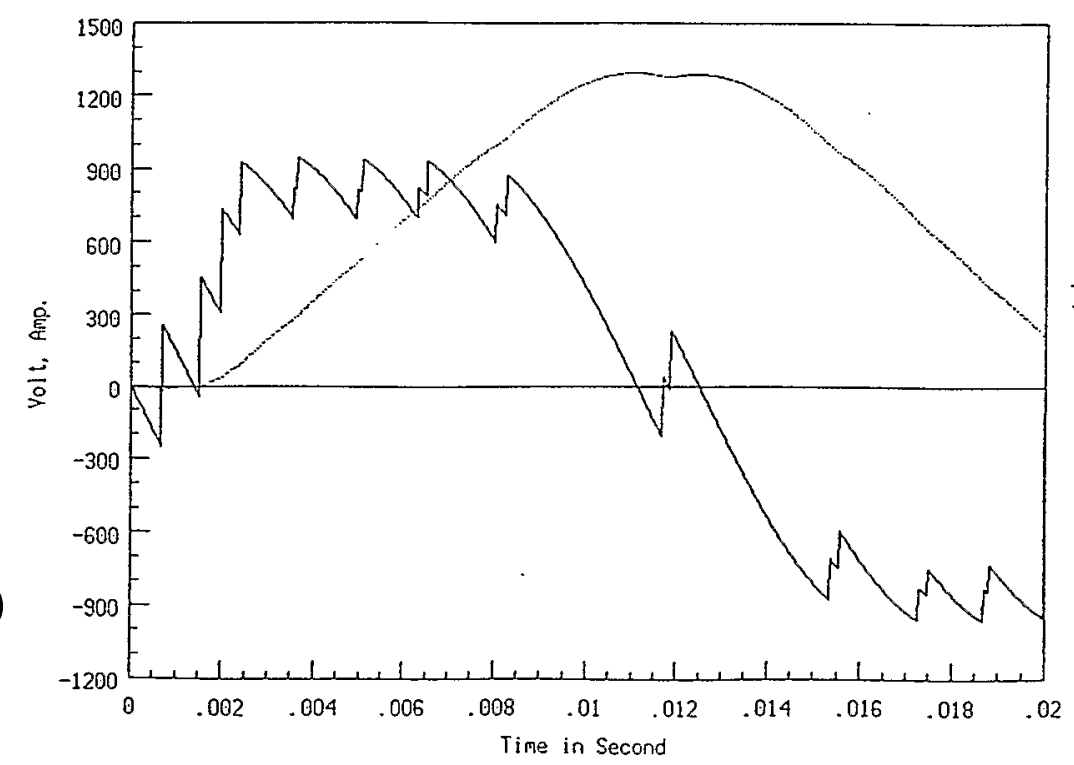

Fig.4.2

24 pulse converter with commutation, higher line inductance

Load Current

Fig.4.3

12 pulse converter with commutation 
$\therefore \ldots$
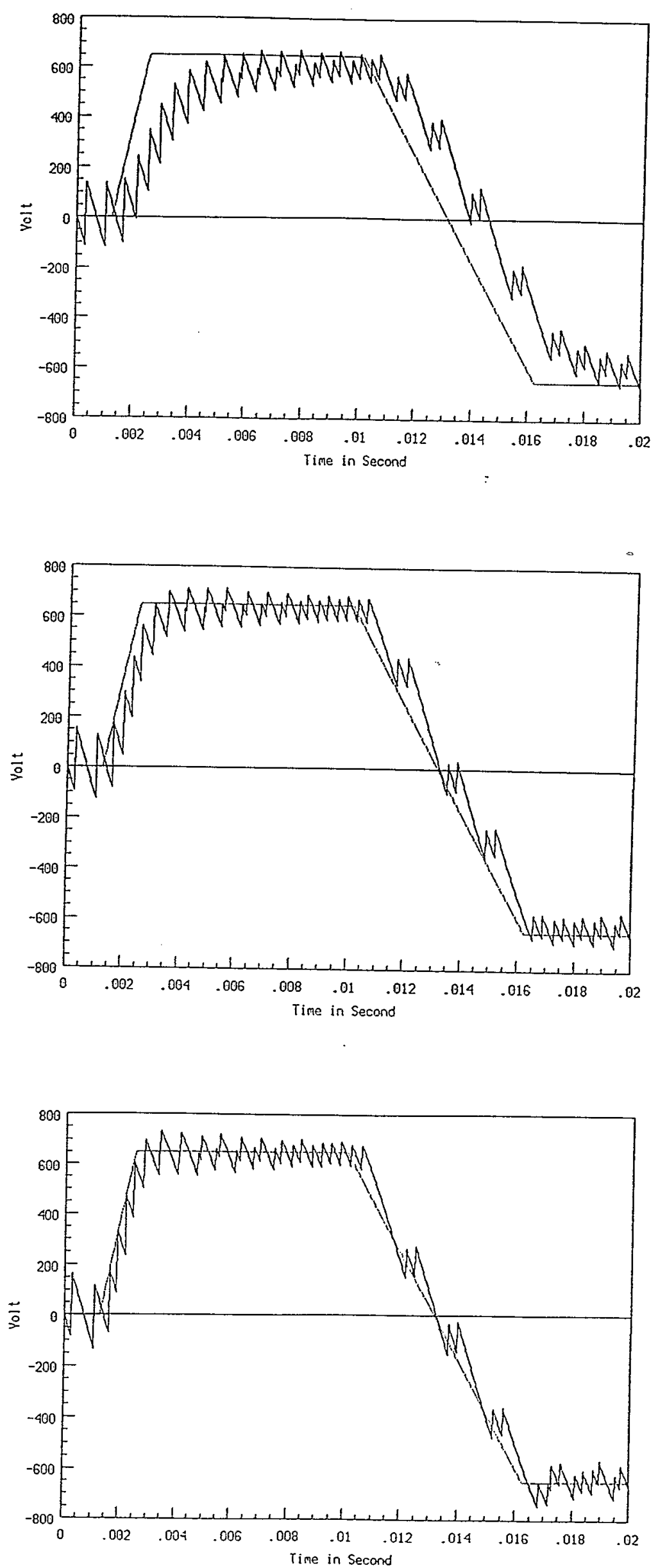

Fig. 5.1

24 pulse regulated converter with commutation, low loop gain

Fig.5.2

24 pulse regulated converter with commutation, medium gain
Fig. 5.3

24 pulse regulated converter with commutation, high loop gain 